\title{
Limits of space-times in five dimensions and their relation to the Segre Types
}

\author{
F. M. Paiva*, M. J. Rebouças ${ }^{\dagger}$ and A. F. F. Teixeira ${ }^{\ddagger}$
}

October 22, 2018

\begin{abstract}
A limiting diagram for the Segre classification in 5-dimensional space-times is obtained, extending a recent work on limits of the energy-momentum tensor in general relativity. Some of Geroch's results on limits of space-times in general relativity are also extended to the context of five-dimensional Kaluza-Klein space-times.
\end{abstract}

PACS numbers: $04.20 . \mathrm{Cv} \quad$ 04.50.+h $\quad$ 04.20.Jb $\quad$ 04.20.-q

\section{Introduction}

In general relativity, it is well known that the curvature tensor can be uniquely decomposed into three irreducible parts, namely the Weyl tensor, the traceless Ricci tensor and the Ricci scalar. Petrov and others [21, 19, 20] have discussed the algebraic classification of the Weyl part, which is known nowadays as Petrov classification. The algebraic classification of the Ricci part, known as the Segre classification, has been discussed by several authors [5] under different viewpoints, and is important, for example, in the characterization of matter

*Departamento de Astrofísica, Observatório Nacional, Rua General José Cristino 77, 20921-400 Rio de Janeiro - RJ, Brazil, INTERNET: FMPAIVA@ON.BR

${ }^{\dagger}$ Centro Brasileiro de Pesquisas Físicas, Rua Dr. Xavier Sigaud 150, 22290-180 Rio de Janeiro - RJ, Brazil, InTERNET: REBOUCAS@CAT.CBPF.BR

${ }^{\ddagger}$ Centro Brasileiro de Pesquisas Físicas, Rua Dr. Xavier Sigaud 150, 22290-180 Rio de Janeiro - RJ, Brazil, INTERNET: TEIXEIRA@NOVELL.CAT.CBPF.BR 
distributions [7, 6, 3, 22, 24], as part of the procedure for checking local equivalence of spacetimes [2, 10, 14, 11, 12, 13], and in the study of limits of non-vacuum solutions of Einstein's field equations 17, 18, 16.

In 1969, Geroch [4] discussed some basic properties of limits of space-times in general relativity (GR). Regarding the algebraic types of the Weyl tensor he showed that the Penrose specialization diagram [19] for the Petrov classification is also a limiting diagram, that is to say, under limiting processes only space-times with the same Petrov type or one of its Penrose specializations can be reached.

In a recent work Paiva et al. [16] have investigated the relations among the Segre types of the Ricci tensor under limiting processes in the framework of general relativity. They have obtained a limiting diagram for the Segre classification of symmetric two-tensors in GR. Among the relevant consequences of their limiting scheme it is worth mentioning that it permits an extension of the coordinate-free approach to limits recently devised [17] (see also [18]).

An essential result for the present article came out in a recent paper by Santos et al. [25] (see also Hall et al. [8]), where they have performed the Segre classification of second order symmetric tensors on five-dimensional (5-D for short) Lorentzian spaces.

In the present paper we build a limiting diagram for the Segre types in five-dimensional space-times, generalizing previous work on this matter [16]. We also extend some of Geroch's results on limits of space-times in general relativity to the context of 5-D Kaluza-Klein-type theories. Although Paiva et al. [17] coordinate-free procedure for finding out limits of spacetimes in GR has not yet been extended to 5-D space-times, the limiting diagram studied in the present work will certainly be relevant to any approach to possible limits of space-times in five dimensions.

Throughout this paper we shall use the concept of limit of a space-time introduced in reference [17] (see also [4, 26]), wherein by a limit of a space-time, broadly speaking, we 
mean a limit of a family of space-times when some free essential parameters are taken to a limit. So, for example, in the one-parameter family of Schwarzschild solutions each member is a Schwarzschild space-time with a specific value for the mass parameter $m$. By space-time we understand a real differential manifold with a metric of Lorentzian signature together with the attendant mathematical structures usually required in physics [9]. Finally we note that although the Ricci tensor is constantly referred to, the results of the following sections apply to any second order real symmetric tensor defined on 5-dimensional Lorentzian spaces.

In the next section we present a brief summary of the main results on Segre classification in 5-D and discuss the minimal and characteristic polynomial types corresponding to the Segre types in 5-D space-times. These results are required for section 3, where we build a limiting diagram for the Segre type in 5-D. In the last section we discuss our main results and their extensions.

\section{Segre Types in 5-D}

The algebraic classification of the Ricci tensor in 5-D space-times can be cast in terms of the eigenvalue problem

$$
\left(R_{b}^{a}-\lambda \delta_{b}^{a}\right) V^{b}=0
$$

where $\lambda$ is a scalar, $V^{b}$ is a vector and the mixed Ricci tensor $R_{b}^{a}$ is looked upon as a linear operator $R: T_{p}(M) \longrightarrow T_{p}(M)$. Here and in what follows $M$ is a real 5 -dimensional spacetime manifold locally endowed with a Lorentzian metric of signature $(+----), T_{p}(M)$ denotes the tangent space to $M$ at a point $p \in M$ and latin indices range from 0 to 4 , unless otherwise stated. Although the matrix $R_{b}^{a}$ is real, the eigenvalues $\lambda$ and the eigenvectors $V^{b}$ are often complex. A mathematical procedure used to classify matrices in such a case is to reduce them through similarity transformations to canonical forms over the complex field. Among the existing canonical forms the Jordan canonical form (JCF) turns out to be the most appropriate for a classification of $R_{b}^{a}$ in 5-D [25]. In the Jordan canonical form, a 
matrix consists of Jordan blocks along the main diagonal. A Jordan block is, for example, a matrix of form

$$
\left[\begin{array}{cccc}
\lambda_{1} & 1 & 0 & 0 \\
0 & \lambda_{1} & 1 & 0 \\
0 & 0 & \lambda_{1} & 1 \\
0 & 0 & 0 & \lambda_{1}
\end{array}\right]
$$

where the equal elements along the main diagonal are the eigenvalue associated to the Jordan block. It is well known that the Jordan canonical form is uniquely defined up to the ordering of the Jordan blocks.

In the Jordan classification two square matrices are said to be equivalent if similarity transformations exist such that they can be brought to the same JCF. The JCF of a matrix gives explicitly its eigenvalues and makes apparent the dimensions of the Jordan blocks. However, for many purposes a somehow coarser classification of a matrix is sufficient. In the Segre classification, for example, the value of the roots of the characteristic equation is not relevant - only dimension of the Jordan blocks and degeneracy of eigenvalues matter. The Segre type is a list $\left[n_{1} n_{2} \cdots n_{r}\right]$ of the dimensions of the Jordan blocks. Equal eigenvalues in distinct blocks are indicated by enclosing the corresponding digits inside round brackets. Thus, for example, in the degenerated Segre type [(31)1] four out of the five eigenvalues are equal; there are three linearly independent eigenvectors, two of which are associated to the Jordan blocks of dimensions 3 and 1, whereas the last one corresponds to the block of dimension 1.

In classifying symmetric tensors in a Lorentzian space-time two refinements to the usual Segre notation are often used. Instead of a digit to denote the dimension of a block with complex eigenvalue a letter is used, and the digit corresponding to a timelike eigenvector is separated from the others by a comma.

As far as 5-D space-times are concerned, due to the Lorentz signature, the real eigenvec- 
tors of $R_{b}^{a}$ may be space-like, null or time-like. For these space-times, it can be shown [25] that some Segre types (as, for example, Segre types [5], [41], [32] and [221]) are not allowed because of the Lorentzian signature of the metric and the symmetry of $R_{a b}$. One also learns from Santos et al. 225 that the possible Segre types of $R_{b}^{a}$ in 5-D Lorentz spaces are:

1. $[1,1111]$ and its degeneracies $[1,11(11)],[(1,1) 111],[1,(11)(11)],[(1,1)(11) 1],[1,1(111)]$, $[(1,11) 11],[(1,1)(111)], \quad[(1,11)(11)], \quad[1,(1111)], \quad[(1,111) 1]$ and $[(1,1111)]$. Type $[(1,1111)]$ implies that $R_{a b}$ is proportional to the metric $g_{a b}$, it is usually referred to as Segre 0.

2. [2111] and its specializations [21(11)], [(21)11], [(21)(11)], [2(111)], [(211)1] and $[(2111)]$. The first digit corresponds to a null eigenvector while the others are associated to space-like eigenvectors.

3. [311] and its degeneracies $[3(11)], \quad[(31) 1]$ and $[(311)]$. Here again the first digit corresponds to a null eigenvector while the others correspond to space-like eigenvectors.

4. $[z \bar{z} 111]$ and its degeneracies $[z \bar{z}(11) 1]$ and $[z \bar{z}(111)]$. Here $z$ and $\bar{z}$ correspond to complex conjugate eigenvectors with complex conjugate eigenvalues. The digits correspond to space-like eigenvectors with real eigenvalues.

We shall now discuss the characteristic and minimal polynomials in connection with Segre types and build a table, which will be important in the derivation of the limiting diagram for the Segre classification of the next section.

Associated to the eigenvalue problem (2.1) one has the determinant

$$
\left|R_{b}^{a}-\lambda \delta_{b}^{a}\right|
$$

which is a polynomial of degree five in $\lambda$, called the characteristic polynomial of $R_{b}^{a}$. The fundamental theorem of algebra [1] ensures that, over the complex field, it can be always 
factorized as

$$
\left(\lambda-\lambda_{1}\right)^{d_{1}}\left(\lambda-\lambda_{2}\right)^{d_{2}} \ldots\left(\lambda-\lambda_{r}\right)^{d_{r}}
$$

where $\lambda_{i}(i=1,2, \cdots, r)$ are the distinct roots of the polynomial (eigenvalues), and $d_{i}$ the corresponding degeneracies. To indicate the characteristic polynomial we shall introduce a new list $\left\{d_{1} d_{2} \cdots d_{r}\right\}$ of eigenvalues degeneracies, hereafter referred to as the type of the characteristic polynomial.

The minimal polynomial can be introduced as follows. Let $P$ be a monic matrix polynomial of degree $n$ in $R_{b}^{a}$, i.e.,

$$
P=R^{n}+c_{n-1} R^{n-1}+c_{n-2} R^{n-2}+\cdots+c_{1} R+c_{0} \delta
$$

where $\delta$ is the identity matrix and $c_{n}$ are, in general, complex numbers. The polynomial $P$ is said to be the minimal polynomial of $R$ if it is the polynomial of lowest degree in $R$ such that $P=0$. It can be shown 27 that the minimal (monic) polynomial is unique and can be factorized as

$$
\left(R-\lambda_{1} \delta\right)^{m_{1}}\left(R-\lambda_{2} \delta\right)^{m_{2}} \cdots,\left(R-\lambda_{r} \delta\right)^{m_{r}}
$$

where $m_{i}$ is the dimension of the Jordan block of highest dimension for each eigenvalue $\lambda_{1}, \lambda_{2}, \cdots, \lambda_{r}$, respectively. We shall denote the minimal polynomial through a third list $\left\|m_{1} m_{2} \cdots m_{r}\right\|$, hereafter referred to as the type of the minimal polynomial.

We can work out now the characteristic and minimal polynomials for each Segre type in 5-D. The power $d_{i}$ of the term corresponding to each eigenvalue $\lambda_{i}$ in the characteristic polynomial is the sum of the dimensions of the Jordan blocks with the same eigenvalue $\lambda_{i}$, whereas in the minimal polynomial the power $m_{i}$ is the dimension of the Jordan block of highest dimension with that eigenvalue. Thus, for example, the Segre types $[(1,111) 1],[(31) 1]$ and $[(211) 1]$ have the same type for the characteristic polynomial, namely type $\{41\}$, while their corresponding minimal polynomials are, respectively, of types $\|11\|,\|31\|$ and $\|21\|$. On the other hand, the Segre types $[3(11)]$ and $[(31) 1]$ have the type $\|31\|$ for the minimal 


\begin{tabular}{|c|c|c|c|c|c|c|c|c|c|c|}
\hline $\begin{array}{l}\mathrm{CP} \rightarrow \\
\mathrm{MP} \downarrow\end{array}$ & $\mid \overline{\mid\{11111\}}$ & 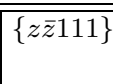 & $\mid \overline{\mid 2111\}}$ & $\overline{\mid \overline{\{21 z \bar{z}\}}}$ & $\overline{\mid \overline{\{221\}}}$ & $\overline{\mid l 311\}}$ & 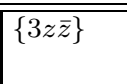 & 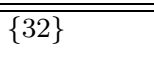 & $\overline{\mid \overline{\{41\}}}$ & $\overline{\mid \overline{\{5\}}}$ \\
\hline$\|111111\|$ & {$[[1,1111]$} & {$[z \bar{z} 111]$} & & & & & & & & \\
\hline$\overline{\mid\|2111\|}$ & & & $\mid[2111]$ & & & & & & & \\
\hline$\|311\|$ & & & & & & {$[311]$} & & & & \\
\hline$\|1111\|$ & & & \begin{tabular}{|l|}
{$[1,11(11)]$} \\
{$[(1,1) 111]$} \\
\end{tabular} & {$[z \bar{z} 1(11)]$} & & & & & & \\
\hline$\|211\|$ & & & & & {$[21(11)]$} & {$[(21) 11]$} & & & & \\
\hline $\mid\|31\|$ & & & & & & & & [3(11)] & $\overline{[(31) 1]}$ & \\
\hline$\|111\|$ & & & & & \begin{tabular}{|l|}
{$[(1,1) 1(11)]$} \\
{$[1,(11)(11)]$}
\end{tabular} & $\begin{array}{l}{[(1,11) 11]} \\
{[1,1(111)]}\end{array}$ & {$[z \bar{z}(111)]$} & & & \\
\hline$\|21\|$ & & & & & & & & $\begin{array}{l}{[2(111)]} \\
{[(21)(11)]}\end{array}$ & {$[(211) 1]$} & \\
\hline$\|3\|$ & & & & & & & & & & {$[(311)]$} \\
\hline$\|11\|$ & & & & & & & & $\begin{array}{l}{[(1,11)(11)]} \\
{[(1,1)(111)]}\end{array}$ & $\begin{array}{l}{[(1,111) 1]} \\
{[1,(1111)]}\end{array}$ & \\
\hline$\|2\|$ & & & & & & & & & & {$[[(2111)]$} \\
\hline$\|1\|$ & & & & & & & & & & {$[(1,1111)]$} \\
\hline
\end{tabular}

Table 1: Characteristic (columns - CP) and minimal (rows - MP) polynomial types corresponding to the Segre types of $R_{b}^{a}$ in 5-D Lorentzian spaces.

polynomial, while the associated characteristic polynomials are, respectively, of types $\{32\}$ and $\{41\}$. We also remark that the Segre types $[2(111)]$ and $[(21)(11)]$ have the same type for both polynomials, namely $\{32\}$ and $\|21\|$.

Table 1 collects together the characteristic (columns - CP) and minimal polynomial (rows - MP) types corresponding to the possible Segre types of a symmetric two-tensor in 5-D Lorentzian spaces. It should be noticed that the characteristic polynomial for the complex Segre types $[z \bar{z} 111],[z \bar{z} 1(11)]$ and $[z \bar{z}(111)]$ have been denoted, respectively, by $\{z \bar{z} 111\}$, $\{21 z \bar{z}\}$ and $\{3 z \bar{z}\}$.

\section{Limiting Diagram for Segre Types in 5-D}

In the study of limits of space-times it is worth noticing that there are some properties that are inherited by all limits of a family of space-times [ [ 7 ]. These properties are called hereditary. Thus, for example, a hereditary property devised by Geroch can be stated as follows: 


\section{Hereditary property:}

Let $T$ be a tensor or scalar field built from the metric and its derivatives. If $T$ is zero for all members of a family of space-times, it is zero for all limits of this family.

Two corollaries of this property are that limits of conformally flat space-times are conformally flat, and that limits of Ricci flat space-times are also vacuum solutions in GR.

In general, the algebraic type of the Weyl tensor is not a hereditary property under limiting processes. Nevertheless, to be at least as specialized as the types in the Penrose specialization diagram for the Petrov classification is 四.

Similarly, although the Segre type of the Ricci tensor is not in general preserved under limiting processes, there exits a limiting diagram for the Segre types in GR, which has been recently discussed [16].

In this section, we shall discuss limiting diagrams for both the characteristic and minimal polynomial types, and combine them to determine a limiting diagram for the Segre types of $R_{b}^{a}$ in 5-D Lorentzian spaces.

Clearly the characteristic (2.3) and the minimal (2.6) polynomials of $R_{b}^{a}$ as well as the eigenvalues are built from the metric and its derivatives [16]. Since they are either scalars or tensors built from the metric and its derivatives (hereafter referred to as Geroch scalars and Geroch tensors), the hereditary property (3.1) can be applied to them.

A limiting diagram for the types of a five degree characteristic polynomial corresponding to the eigenvalue problem (2.1) can now be constructed. We first note that as at each degeneracy one Geroch scalar (the difference between two roots of the characteristic polynomial) vanishes, by the hereditary property (3.1), under a limiting process, the degeneracy of the characteristic polynomial either increases or remains the same. Besides, the real and imaginary parts of complex roots are also Geroch scalars. Therefore, Segre types with real roots 
cannot have as a limit a Segre type with a complex root. Further, since complex roots can occur only in complex conjugate pairs, under a limiting process they either remain complex or become a pair of degenerate real roots. These results can be collected together in the limiting diagram for the characteristic polynomial shown in figure 1. For the sake of simplicity, in the limiting diagrams in this paper, we do not draw arrows between types whenever a compound limit exists. Thus, in figure 1, e.g., the limits $\{11111\} \rightarrow\{2111\} \rightarrow\{311\} \rightarrow\{32\}$ imply that the limit $\{11111\} \rightarrow\{32\}$ is allowed.

A limiting diagram for the types of a five degree minimal polynomial of the Ricci tensor can be constructed as follows. According to the hereditary property (3.1), the minimal polynomial of a family of space-times is zero for all limits of this family. Therefore, under limiting processes the degree of the minimal polynomial either decreases or remains the same. Besides, from the limiting diagram for the characteristic polynomial in figure — we notice that also the number $r$ of distinct eigenvalues either decreases or remains the same. Taking into account these properties we can work out the limiting diagram for the minimal polynomial shown in figure 2, where the columns correspond to the same degree $m_{1}+m_{2}+\cdots+m_{r}$ of the minimal polynomial, and the rows correspond to the same number $r$ of distinct eigenvalues. It should be noticed that although we could also have distinguished complex from real roots in the minimal polynomial diagram, for our purpose in this paper it can be verified that no useful information would arise.

From the limiting diagrams for the characteristic and minimal polynomials in figures 1 and 2, we substitute for each type of the characteristic and minimal polynomials the corresponding Segre types taken from table 1. This gives rise to the two limiting diagrams given in figures 3 and 4 , respectively. We have not taken into account the character of the eigenvectors. Thus, for example, we represent the Segre types $[(1,1) 111]$ and $[1,11(11)]$ by the set-type $[(11) 111]$ and the Segre types $[(1,11)(11)]$ and $[(1,1)(111)]$ by the set-type $[(111)(11)]$. The set-types $[(11) 1(11)]$ and [(111)11] are similarly introduced. 


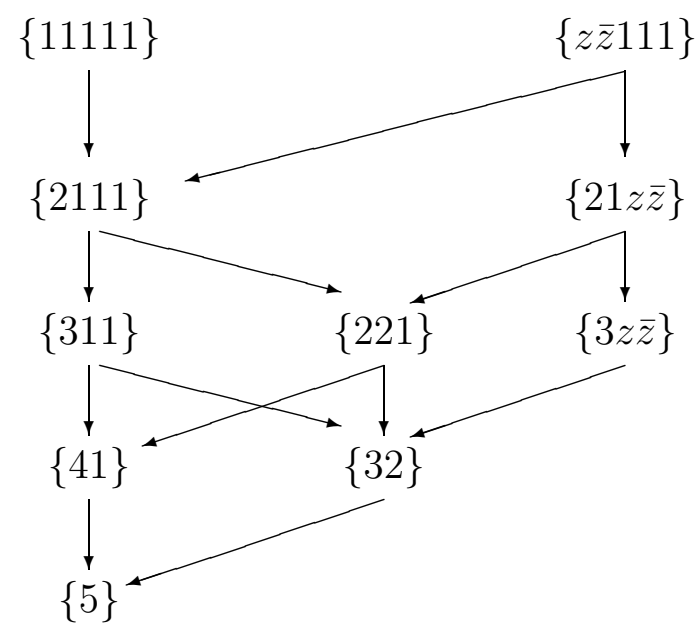

Figure 1: Limiting diagram for the characteristic polynomial in 5-D Lorentzian spaces.

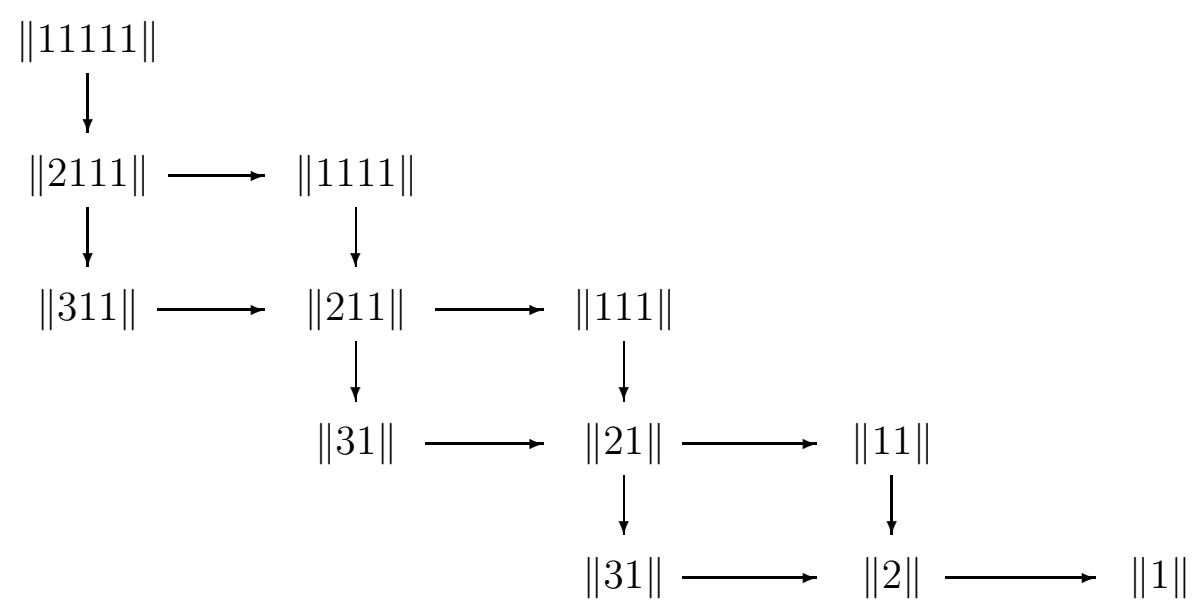

Figure 2: Limiting diagram for the minimal polynomial in 5-D Lorentzian spaces. The types $\|221\|,\|32\|,\|22\|,\|4\|$ and $\|5\|$ are not shown since they do not correspond to any Segre type (see table (1). 
We can now collect together the information of the limiting diagrams of figures 3 and 4 , to finally draw a limiting diagram for the Segre types in 5-D, shown in figure 5. Thus, for

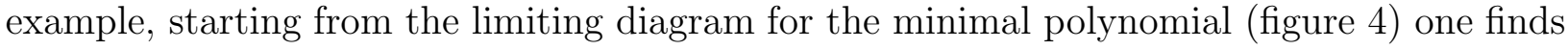

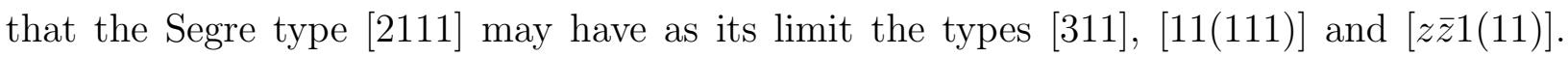
However, from the diagram for the characteristic polynomial shown in figure 1 one finds that the Segre type [2111] cannot have the type $[z \bar{z} 1(11)]$ as its limit. So, we have only two arrows starting from the Segre type [2111], as it has been drawn in diagram 5. Although the other arrows in figure 5 can be similarly determined, we shall not discuss them here for the sake of brevity.

To close this section we remark that again in the limiting diagram shown in figure 5 the character of the eigenvectors is not taken into account. We shall return to this point in the next section.

\section{Conclusion}

In this work we have constructed a limiting diagram for the Segre classification of a second order symmetric two-tensor defined on 5-D Lorentzian spaces (figure 5). To achieve this goal we have essentially used the hereditary property (3.1) together with the limiting diagrams for the characteristic and minimal polynomial types, which we have worked out in section 3 .

Improvements of the limiting diagram that we have presented in this article can still be tackled. A first refinement would arise by taking into account the character of the eigenvectors. To take into account the types which differ by the character of the eigenvectors one has firstly to separate the set-types into its two members, and check whether one of these members can have the other as its limits and vice-versa. Secondly, one needs to find out whether the Segre types which can have as limit one set-type can have a limit both members of the set. Finally, one ought to examine whether the Segre types which can be a limit of one set-type can be a limit of each member of the corresponding Segre set-type. 


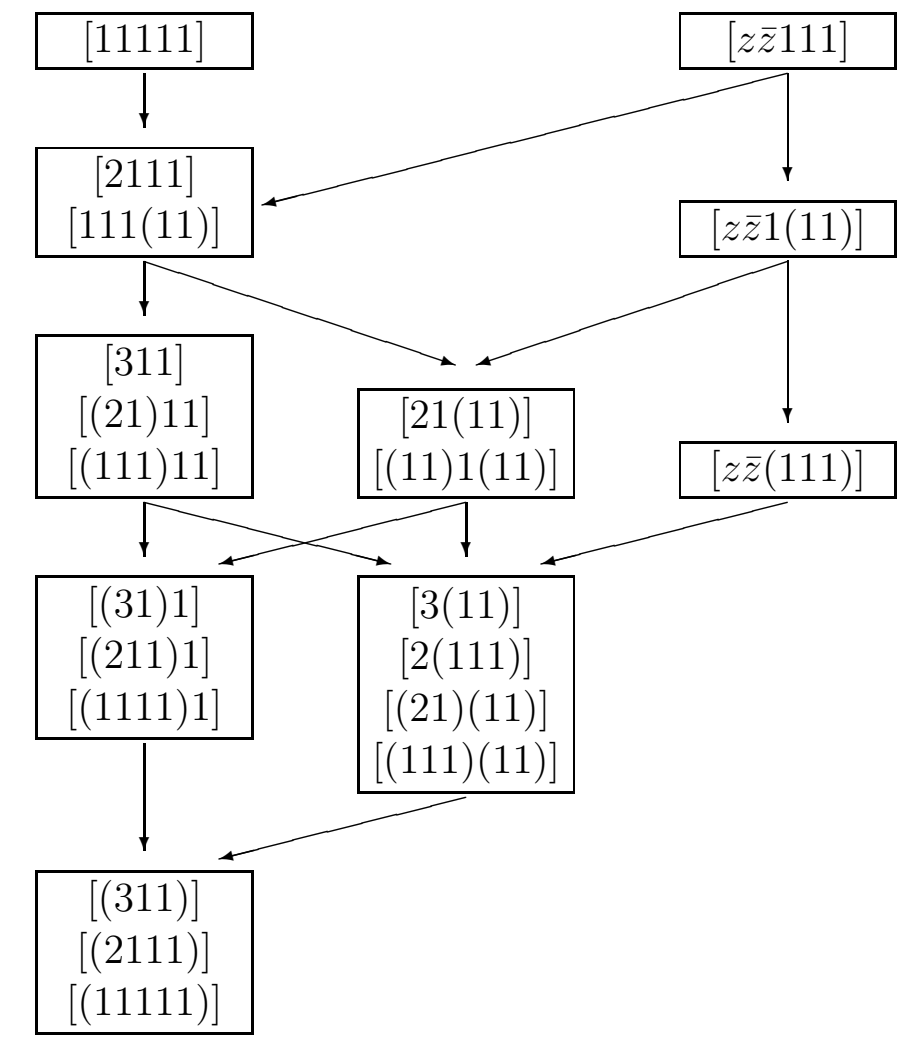

Figure 3: Diagram for the limits of Segre types of $R_{b}^{a}$ in 5-D Lorentzian spaces according to the types of the characteristic polynomial. 


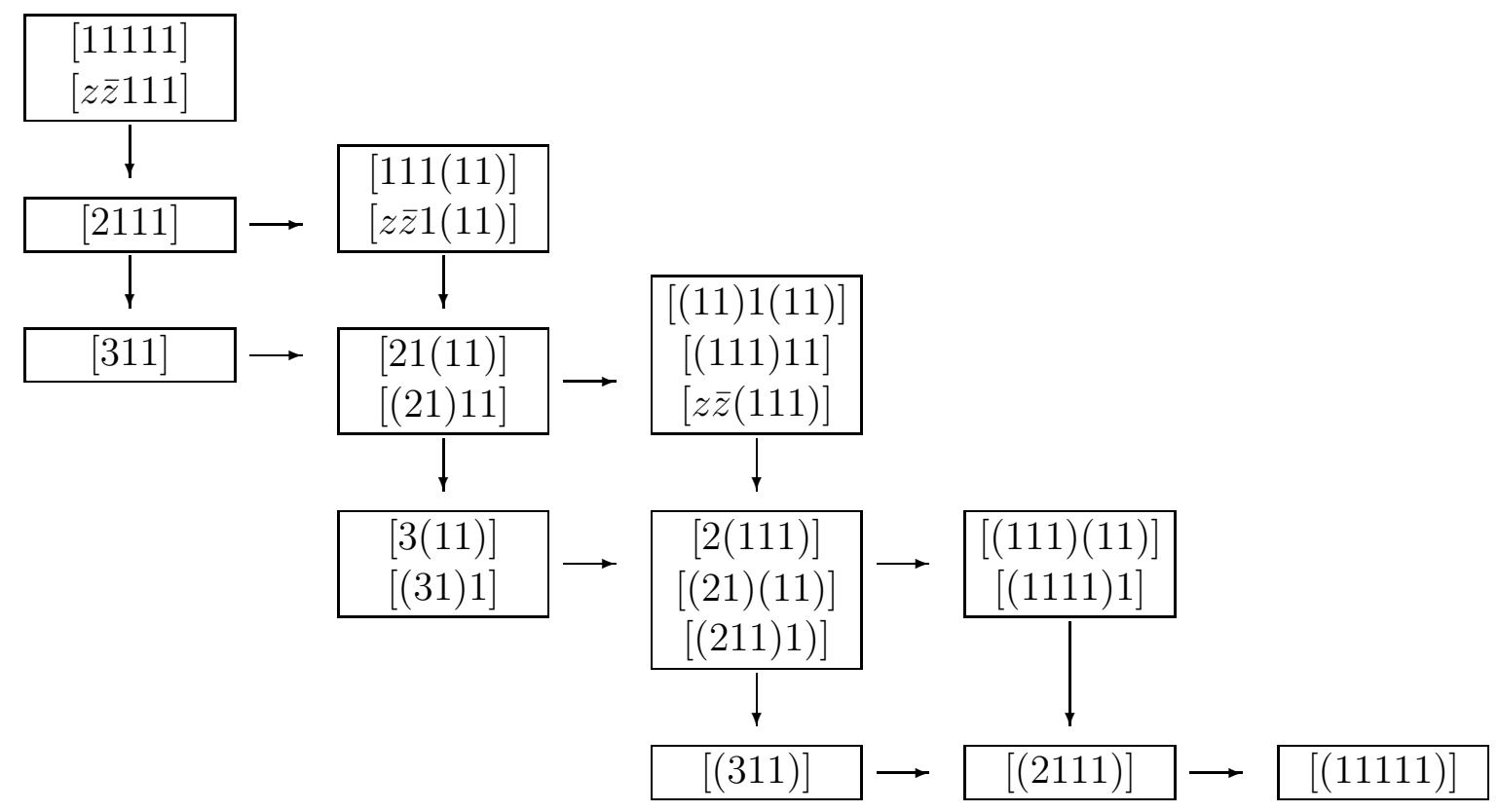

Figure 4: Diagram for the limits of Segre types of $R_{b}^{a}$ in 5-D Lorentzian spaces according to the type of the minimal polynomial.

Perhaps most of these checkings can be made simply by extending to 5 -D space-times the hereditary properties discussed in the context of GR [16]. A second refinement of the limiting diagram shown in figure 5 can be made by figuring out a criterion for separating the Segre types $[2(111)]$ and $[(21)(11)]$, which have the same type for both characteristic and minimal polynomials. A third improvement of the limiting diagram given in figure 5 might arise if besides the type of the characteristic and minimal polynomials one considers the values of their roots.

The limiting diagrams of the Petrov and the Segre classification play a fundamental role in the study of limits of space-times in general relativity [17, 18, 15, as briefly discussed in the introduction. Although the coordinate-free technique for finding out limits of space-times in GR [17] have not yet been extended to 5-D space-times, the limiting diagram studied in the present work will certainly be applicable to any coordinate-free approach to possible limits of non-vacuum space-times in five dimensions. 


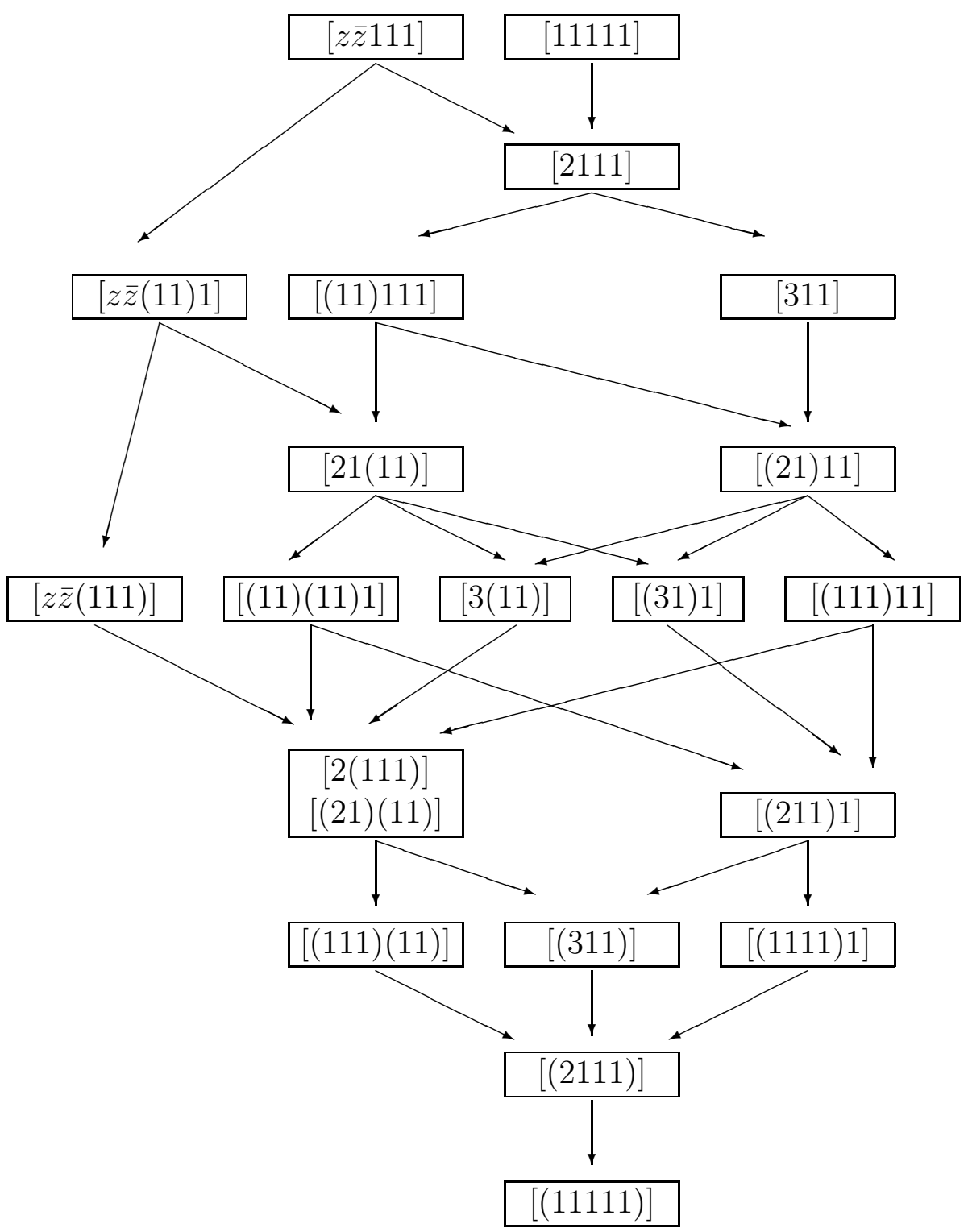

Figure 5: Diagram for the limits of the Segre types of $R_{b}^{a}$ in 5-D Lorentzian spaces. 


\section{Acknowledgment}

The authors gratefully acknowledge financial assistance from CNPq.

\section{References}

[1] Cajori, F. (1969), An Introduction to the Theory of Equations, Dover Publications, Inc, New York.

[2] Cartan, E. (1951), Leçons sur la Géométrie des Éspaces de Riemann, Gauthier-Villars, Paris. Reprinted, Éditions Jacques Gabay, Paris (1988). English translation by J. Glazebrook, Math. Sci. Press, Brookline (1983).

[3] Ferrando, J.J., Morales, J.A. \& Portilla, M. (1990), Two-Perfect Fluid Interpretation of an Energy Tensor, Gen. Rel. Grav. 22, 1021-1032.

[4] Geroch, R. (1969), Limits of Space-times, Commun. Math. Phys. 13, 180-193.

[5] Hall, G.S. (1984), The Classification of Second Order Symmetric Tensors in General Relativity Theory, Diff. Geom. 12, 53-73. This reference contains an extensive bibliography on the classification of the Ricci tensor on 4-dimensional Space-times.

[6] Hall, G.S. (1984), The Structure of the Energy-momentum Tensor in General Relativity, Arab. J. Sci. Eng. 9, 87-96.

[7] Hall, G.S. \& Negm, D.A. (1986), Physical Structure of the Energy-momentum Tensor in General Relativity, Int. J. Theor. Phys. 25, 405-423.

[8] Hall, G.S., Rebouças, M.J., Santos, J. \& Teixeira, A.F.F. (1996), On the Algebraic Structure of Second Order Symmetric Tensors in 5-Dimensional Space-times, Gen. Rel. Grav. 28, in press. 
[9] Hawking, S.W. \& Ellis, G.F.R. (1973), The Large Scale Structure of Space-time, Cambridge U. P., Cambridge.

[10] Karlhede, A. (1980), A Review of the Geometrical Equivalence of Metrics in General Relativity, Gen. Rel. Grav. 12, 693-707.

[11] MacCallum, M.A.H. (1983), "Classifying Metrics in Theory and Practice", in Unified Field Theory in More Than 4 Dimensions, Including Exact Solutions, edited by V. de Sabbata and E. Schmutzer. World Scientific Publishing Co., Singapore.

[12] MacCallum, M.A.H. (1984), "Algebraic Computing in General Relativity", in Classical General Relativity, edited by W.B. Bonnor, J.N. Islam and M.A.H. MacCallum. Cambridge U. P., Cambridge.

[13] MacCallum, M.A.H. (1991), "Computer-aided Classification of Exact Solutions in General Gelativity", in General Relativity and Gravitational Physics (9th Italian Conference), edited by R. Cianci, R. de Ritis, M. Francaviglia, G. Marmo, C. Rubano and P. Scudellaro, pp. 318-337, World Scientific, Singapore.

[14] MacCallum, M.A.H. \& Skea, J.E.F. (1994), "SheEP: A Computer Algebra System for General Relativity", in Algebraic Computing in General Relativity, Lecture Notes from the First Brazilian School on Computer Algebra, Vol. II, edited by M.J. Rebouças and W.L. Roque. Oxford U. P., Oxford; and references therein.

[15] Paiva, F.M. (1993), Limites de Espaços-tempos em Gravitação, Ph. D. thesis, Brazilian Center for Physics Research (CBPF), Rio de Janeiro.

[16] Paiva, F.M., Rebouças, M.J., Hall, G.S. \& MacCallum, M.A.H. (1996), Limits of the Energy-momentum Tensor in General Relativity, to be submitted. 
[17] Paiva, F.M., Rebouças, M.J. \& MacCallum, M.A.H. (1993), On Limits of Space-times - a Coordinate-free Approach, Class. Quantum Grav. 10, 1165-1178.

[18] Paiva, F.M. \& Romero, C. (1993), The Limits of Brans-Dicke Space-times: a Coordinate-free Approach, Gen. Rel. Grav. 25, 1305-1317.

[19] Penrose, R. (1960), A Spinor Approach to General Relativity, Ann. Phys. NY 10, 171201.

[20] Penrose, R. \& Rindler, W. (1986), Spinors and Space-time, vol. 2, Cambridge U. P., Cambridge.

[21] Petrov, A.Z. (1969), Einstein Spaces, first English edition, Pergamon Press.

[22] Rebouças, M.J. \& Teixeira, A.F.F. (1991), Einstein Universe as a Moving Fluid Cosmology, J. Math. Phys. 32, 1861-1865.

[23] Rebouças, M.J. \& Teixeira, A.F.F. (1992), Homogeneous Space-times with Seven Isometries, J. Math. Phys. 33, 2855-2862.

[24] Santos, J., Rebouças, M.J. \& Teixeira, A.F.F. (1993), Classification of Scalar Fields in General Relativity, J. Math. Phys. 34, 186-192.

[25] Santos, J., Rebouças, M.J. \& Teixeira, A.F.F. (1995), Classification of Second Order Symmetric Tensors in 5-dimensional Kaluza-Klein-type Theories, J. Math. Phys. 36, 3074-3084.

[26] Schmidt, H.-J. (1987), On Geroch's Limit of Space-times and its Relation to a New Topology in the Space of Lie Groups, J. Math. Phys. 28, 1928-1264.

[27] Wilkinson, J.H. (1965), The Algebraic Eigenvalue Problem, Clarendon Press, Oxford. 